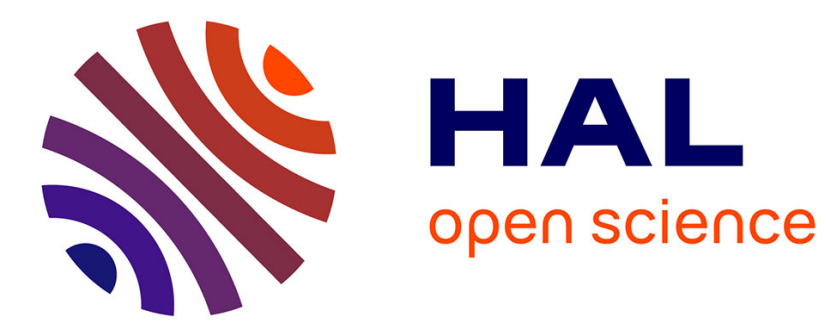

\title{
Les femmes artistes sous presse
}

Charlotte Foucher Zarmanian

\section{To cite this version:}

Charlotte Foucher Zarmanian. Les femmes artistes sous presse: Les créatrices vues par les femmes critiques d'art dans la presse féminine et féministe en France autour de 1900. Sociétés \& Représentations, 2015. hal-03020187

\section{HAL Id: hal-03020187 https://hal.science/hal-03020187}

Submitted on 23 Nov 2020

HAL is a multi-disciplinary open access archive for the deposit and dissemination of scientific research documents, whether they are published or not. The documents may come from teaching and research institutions in France or abroad, or from public or private research centers.
L'archive ouverte pluridisciplinaire HAL, est destinée au dépôt et à la diffusion de documents scientifiques de niveau recherche, publiés ou non, émanant des établissements d'enseignement et de recherche français ou étrangers, des laboratoires publics ou privés. 


\section{LES FEMMES ARTISTES SOUS PRESSE}

Les créatrices vues par les femmes critiques d'art dans la presse féminine et féministe en France autour de 1900

\section{Charlotte Foucher Zarmanian}

Éditions de la Sorbonne | "Sociétés \& Représentations 》

2015/2 N 40 | pages 111 à 127

ISSN 1262-2966

Article disponible en ligne à l'adresse :

https://www.cairn.info/revue-societes-et-representations-2015-2-page-111.htm

\section{(cc) BY-NC-ND}




\section{Les femmes artistes sous presse Les créatrices vues par les femmes critiques d'art dans la presse féminine et féministe en France autour de 1900}

La production critique des femmes sur l'art au XIX ${ }^{\mathrm{e}}$ siècle est devenue depuis plus de trente ans un véritable objet d'étude. À l'origine de ce mouvement, l'ouvrage Women as Interpreters of the Visual Arts, 1820-1979 dirigé par Claire Richter Sherman, s'intéresse à des femmes - principalement britanniques et américaines - qui ont été non seulement critiques d'art, libraires, traductrices, mais également historiennes de l'art, archéologues et conservatrices du patrimoine ${ }^{1}$. Trois ans plus tard, en 1984, c'est au tour de Vivian Cameron de consacrer un article à deux femmes critiques d'art françaises qui se sont opposées, lors de la Révolution française, aux écrits de leurs homologues masculins et à un certain phallocentrisme en $\operatorname{art}^{2}$. Ces deux chercheuses sont parvenues à inaugurer, grâce à leurs publications, un large champ de recherches en histoire de l'art permettant la redécouverte de personnalités méconnues et de discours oubliés.

Il faudra toutefois attendre les années 2000 pour dépasser la simple exhumation et assister à une meilleure prise en compte analytique, théorique et historiographique de ce champ au bénéfice d'une plus large intégration des études féministes et de genre en histoire de l'art et en histoire littéraire ${ }^{3}$. Publié

1. Claire Richter Sherman (dir.), Women as Interpreters of the Visual Arts, 1820-1979, Westport/Londres, Greenwood Press, 1981, 487 p.

2. Vivian Cameron, "Two 18th Century French Art Critics », Women's Art Journal, vol. 5, n 1, 1984, p. 8-11.

3. Katy Deepwell, " Défier l'indifférence à la différence : les paradoxes de la critique d'art féministe », dans Pierre-Henry Frangne et Jean-Marc Poinsot (dir.), L'Invention de la critique d'art, actes du colloque international tenu à l'université Rennes 2 les 24 et 25 juin 1999, Rennes, Presses universitaires de Rennes, 2002, p. 191-205; Christine Planté, Marie-Ève Thérenty, « Masculin/Féminin dans la presse du xIx siècle. Le genre de la critique », dans Muriel Andrin, Laurence Brogniez, Alexia Creusen, Amélie Favry, Vanessa 
en 1989, l'essai d'Anne Higonnet, "Situation critique de la féminité ", n'est certainement pas étranger à ce nouvel intérêt ${ }^{4}$. Dépassant la dichotomie essentialiste qui consistait à qualifier l'art produit par les femmes de "féminin " contre l'art " masculin " produit par les hommes, Higonnet examine les versants et fonctions idéologiques du discours critique sur l'art en s'intéressant plus largement à son iconographie et à son style.

Les travaux publiés par la suite restent redevables à cette autre lecture du discours critique, en posant dorénavant la question d'une spécificité du regard des femmes dans ces milieux savants dominés par les hommes ou encore de leur légitimité en tant qu'auteures. Ainsi, Laurence Brogniez développe notamment des analyses sur les jeux identitaires, les stratégies d'écritures et les supports privilégiés, afin d'appréhender la critique d'art comme une possibilité pour les femmes de questionner leur propre condition et leur perception d'ellesmêmes ${ }^{5}$. Plus récemment, deux ouvrages importants se sont intéressés à la production théorique des femmes sur l'art au XviII ${ }^{\mathrm{e}}$ siècle et au XIX ${ }^{\mathrm{e}}$ siècle. Le premier, Plumes et pinceaux. Discours des femmes sur l'art en Europe (1750-1850), analyse en deux volumes les réflexions d'auteures sur l'art en les intégrant aux débats esthétiques contemporains ${ }^{6}$. À partir de divers textes et supports (correspondances, traités pédagogiques, écrits de restauration, récits de voyage, comptes rendus de Salons...), et à l'appui d'une multiplicité de parcours, ce

Gemis (dir.), Femmes et critique(s). Lettres, Arts, Cinéma, Namur, Presses universitaires de Namur, 2009, p. 15-30; Texte \& Image, $n^{\circ}$ 1, 2011, Marianne Camus, Valérie Dupont (dir.), Les Femmes parlent d'art, http://revuesshs.u-bourgogne.fr/texte\&image/sommaire.php?id=27, consulté le 22 octobre 2014 ; Martine Reid (dir.), Les Femmes dans la critique et l'histoire littéraire, Paris, Honoré Champion, 2011, 212 p.

4. Anne Higonnet, "Situation critique de la féminité », dans Jean-Paul Bouillon (dir.), La Critique d'art en France (1850-1900), actes du colloque de Clermont-Ferrand, 25-27 mai 1987, université de Saint-Etienne, Centre interdisciplinaire d'études et de recherches sur l'expression contemporaine (CIEREC), 1989, p. 121133. Cet essai a fait l'objet d'un remaniement et d'une traduction en anglais sous le titre : "Imaging Gender ", dans Michael R. Orwicz (dir.), Art Criticism and its Institutions in Nineteenth-Century France, Manchester, Manchester University Press, 1994, p. 146-161.

5. Laurence Brogniez, "Claude Vignon, une femme "surexposée" sous le Second Empire », dans David Baguley (dir.), Les Arts et la littérature sous le Second Empire, actes du colloque international organisé par le French Department de I'université de Durham, 18-20 avril 2002, Durham, University of Durham Modern Languages Series, 2003, p. 149-165 ; Laurence Brogniez, "La critique d'art au féminin au xIx siècle : Marc de Montifaud ou le discours (dé)voilé ", Art \& Fact, n²4, 2005, Femmes et créations, p. 15-23; Laurence Brogniez, "Les femmes au Salon : proposition pour une étude de la critique d'art féminine au xix siècle », dans Christine Planté (dir.), Les Lieux littéraires. Féminin/Masculin. Écritures et représentations. Corpus collectifs, n 7-8, 2005, p. 113-126; Muriel Andrin, Laurence Brogniez, Alexia Creusen, Amélie Favry, Vanessa Gemis (dir.), Femmes et critique(s), op. cit.

6. Melissa Hyde, Mechthild Fend, Anne Lafont (dir.), Plumes et pinceaux. Discours de femmes sur l'art en Europe (1750-1850), vol. 1, Dijon, Presses du réel, 2012, 333 p. ; Anne Lafont (dir.), avec la collaboration de Charlotte Foucher et Amandine Gorse, Plumes et pinceaux. Discours de femmes sur l'art en Europe (1750-1850) - Anthologie, vol. 2, Dijon, Presses du réel, 2012, 549 p. 
livre traite d'une époque déterminante pour l'émergence, la démocratisation et la professionnalisation de la critique d'art, laquelle avait été peu traitée pour le cas féminin. Le second ouvrage, Women Art Critics in Nineteenth-Century France. Vanishing Acts, dirigé par Wendelin Guentner, professeure de littérature française à l'université d'Iowa, balaie un large XIX ${ }^{\mathrm{e}}$ siècle, de l'époque révolutionnaire jusqu'aux années $1900^{7}$. Affichant un parti pris délibérément pluridisciplinaire mêlant histoire de l'art et littérature, il met en avant le contexte culturel dans lequel les femmes critiques d'art évoluent, tout en prêtant attention à leurs modalités scripturales et à leurs stratégies identitaires pour se faire une place dans ce milieu à dominante masculine.

Guentner reconnaît toutefois, en conclusion de son ouvrage, l'impossibilité de l'exhaustivité d'un tel sujet et invite à explorer d'autres pistes de recherches qui mériteraient, selon elle, d'être plus largement approfondies. Celle de la critique d'art délivrée dans la presse féminine de la seconde moitié du $\mathrm{XIX}^{\mathrm{e}}$ siècle retient particulièrement son attention ${ }^{8}$. Ce sujet a déjà pu bénéficier de l'intéressant ouvrage de Meaghan Clarke qui analysait, en 2005, la place occupée par les femmes dans la presse artistique de l'Angleterre victorienne ${ }^{9}$. Dans ce livre, Clarke révèle une multitude de postures, d'attitudes et de points de vue allant à l'encontre d'un prétendu particularisme des femmes dans les discours sur l'art. Elle examine notamment les liens que certaines d'entre elles entretiennent avec les milieux féministes et démontre aussi combien le discours artistique permet plus largement de faire progresser les combats politiques, sociaux et idéologiques en faveur des femmes ${ }^{10}$.

Comme dans l'Angleterre victorienne, le contexte français autour de 1900 semble favoriser cette émancipation des femmes dans les mondes de l'art. La seconde moitié du XIX ${ }^{\mathrm{e}}$ siècle est en effet marquée, non seulement par la professionnalisation de la critique d'art, accompagnée par la loi sur la liberté de la presse de 1881 et la création du Syndicat de la presse artistique française en 1899, mais également par la construction d'un enseignement secondaire

7. Wendelin Guentner (dir.), Women Art Critics in Nineteenth-Century France. Vanishing Acts, Newark, University of Delaware Press, 2013, 366 p.

8. Ibid., p. 273.

9. Meaghan Clarke, Critical Voices. Women and Art Criticism in Britain, 1880-1905, Aldershot, Ashgate, 2005, 214 p.

10. Pour une étude plus approfondie de la place des femmes dans le journalisme fin-de-siècle britannique, voir Elizabeth F. Gray (dir.), Women in Journalism in the Fin de Siècle: Making a Name for Herself, New York, Palgrave Macmillan, 2012, 259 p. 
pour les jeunes filles dans lequel l'art est au programme ${ }^{11}$. Ces différents événements contribuent à la féminisation des discours et pensées critiques sur l'art. Deux institutions, également, ouvrent autour de 1900 leurs portes aux femmes : l'École du Louvre en 1882, première école spécialisée dans l'enseignement de l'histoire de l'art que fréquentent de nombreuses femmes ${ }^{12}$, et l'École des beaux-arts de Paris qui permet officiellement en 1897 aux femmes de recevoir un enseignement pratique et théorique en $\operatorname{art}^{13}$.

Souhaitant confronter cette situation politique, sociale et institutionnelle plutôt propice aux femmes à une étude des discours et des représentations, nous verrons comment les femmes critiques d'art traitent du sort de leurs consœurs créatrices. Vont-elles participer à la conquête de nouveaux droits, contribuer à une plus grande visibilité de leur statut, ou au contraire reconduire stéréotypes et clichés ? S'intéresser aux femmes engendre-t-il obligatoirement une pensée féministe, ou peut-il être considéré comme un moyen stratégique d'entrisme pour les critiques d'art dans ces milieux journalistiques ? De la polarisation sur un corpus d'œuvres d'art peu abordé par le discours critique dominant à l'affirmation plus ou moins militante d'un point de vue strictement "féminin " sur l'art, il s'agira d'examiner la multiplicité des pratiques et dispositifs d'écriture mobilisée par ces auteures dans la presse féminine et féministe autour de 1900 tout en prêtant attention à la marge des perceptions et définitions possibles au sein des féminismes.

\section{Pluralismes et divergences des féminismes}

Parallèlement à la prolifération des discours psychopathologiques défendant l'incompatibilité des femmes à pouvoir prétendre à la fois à la création et à la procréation, la figure de l'artiste épanouie et équilibrée naît également autour de 1900, dans un contexte où l'on ne formule plus strictement l'émancipation féminine comme un vecteur d'angoisse mais où l'on s'interroge aussi sur le bonheur d'être une femme émancipée ${ }^{14}$.

11. Antoine Prost, «Inférieur ou novateur ? L'enseignement secondaire des jeunes filles (1880-1887) », Histoire de l'éducation, n 115-116, 2007, p. 149-169.

12. Lyne Therrien, L'Histoire de l'art en France. Genèse d'une discipline universitaire, Paris, CTHS, 1998, p. $169-216$

13. Marina Sauer, L'Entrée des femmes à l'École des beaux-arts, 1880-1923, Paris, ENSBA, 1990, 89 p.

14. Voir Maurice de Waleffe, "La Femme artiste est-elle heureuse ? ", Le Figaro, 9 décembre 1901, p. 1. Sur I'opposition création/procréation, voir Charlotte Foucher, « Le Péril bleu. Images psychopathologiques de la femme artiste au passage du siècle », actes du colloque " Images de l'artiste », sous la direction de 
Le support qui relaie, à l'envi, la possible conciliation entre vie professionnelle et vie personnelle des femmes artistes est bien celle de la presse féminine et féministe, en nette progression à la fin du siècle. Si la presse féminine, représentée par des magazines illustrés tels que La Vie heureuse (1892) et Femina (1901), se multiplie à la Belle Époque, la presse féministe prend véritablement son essor sous la Troisième République et s'attache à soutenir la revendication des droits politiques, civils et sociaux des femmes (droit de vote, création de syndicats... $)^{15}$.

Cependant, on est en droit de s'interroger, face à la multitude des périodiques $^{16}$, à l'hétérogénéité des supports et de leurs vocations, à la diversité des équipes rédactionnelles ${ }^{17}$, et à celle plus disparate encore du lectorat ${ }^{18}$, sur le problème de la ligne et de la cohérence idéologique de tous ces discours, et donc sur la difficulté à fixer une image précise de "la " femme artiste à cette époque à travers les textes critiques.

\section{Femmes artistes dans la presse féministe : une émancipation revendiquée}

Le tournant du siècle est marqué par la diffusion massive de périodiques ouvertement militants qui, dans une veine égalitariste, saluent la libération

Éric Darragon et Bertrand Tillier, INHA/Paris 1 HiCSA, dans Territoires contemporains, revue en ligne du Centre Georges-Chevrier, université de Bourgogne, 2012, http://tristan.u-bourgogne.fr/CGC/publications/ image_artiste/Charlotte_Foucher.html, consulté le 22 octobre 2014.

15. La presse féminine (Journal des Dames, Le Cabinet des Modes, ou encore Le Journal des Dames et des modes) connaît une nette augmentation, avec 63 nouveaux titres, entre 1818 et 1847, puis avec 34 nouveaux titres, entre 1849 et la fin du Second Empire. De 1870 à 1914, une trentaine de nouvelles créations, illustrées cette fois-ci, voient le jour. La première revue féministe, La Femme libre, paraît en 1832 mais ce type de presse engagée prend véritablement son essor sous la Troisième République avec des revues telles que La Femme dans la famille et dans la société (1881-1882) remplacée par La Femme et I'enfant (1884-1887) de Louise Koppe, La Citoyenne d'Hubertine Auclert (1881-1891), La Femme de I'avenir (1888-1901), La Fronde de Marguerite Durand (1897-1914), L'Abeille de Pauline Savari (1901-1902), La Suffragiste de Madeleine Pelletier (1911-1914). Parmi les quelques études qui existent aujourd'hui sur cette presse, voir Li Dzeh-Den, La Presse féministe en France de 1869 à 1914, thèse de doctorat en lettres, université de Paris, Paris, Librairie L. Rodstein, 1934, 236 p. ; Évelyne Sullerot, Histoire de la presse féminine en France, des origines à 1848, Paris, Armand Colin, 1966, 227 p. ; Gilles Feyel, « La Presse féminine au xIx siècle (1797-1914) », dans Hélène Eck et Claire Blandin (dir.), La Vie des femmes. La presse féminine aux $x i x^{e}$ et $x x^{e}$ siècles, Paris, Éditions Panthéon-Assas, 2010, p. 31-47.

16. Nous avons peu d'informations au sujet des tirages de Femina et La Vie heureuse qui se vendaient plutôt bien. En revanche, nous savons que le journal La Fronde était par exemple tiré en 1898 à 50000 exemplaires.

17. Si la rédaction de La Fronde est exclusivement composée de femmes, celle de Femina est majoritairement constituée d'hommes (son directeur est Pierre Lafitte).

18. Marguerite Durand souhaitait que La Fronde soit «l'organe de toutes les femmes françaises », alors que Femina et La Vie heureuse s'adressaient en priorité aux "femmes du monde », issues de milieux bourgeois et privilégiés. 
des femmes artistes. En témoigne un article paru dans La Fronde où l'écrivaine Marcelle Tinayre renverse le point de vue dominant et détourne, de manière ironique et provocante, le discours canonique en reléguant la femme osant s'émanciper au rang d'une dévergondée :

On en a conclu que toute femme capable d'exprimer sa pensée ou son émotion sous une forme artistique était forcément une dévergondée. On ne s'est pas demandé si cette singulière émigration des femmes artistes vers la liberté, n’avait pas d'autres causes et un autre but que le dévergondage ${ }^{19}$.

Ce revirement de point de vue dans le discours est symptomatique du ton de ces nouveaux journaux féministes qui ne sont plus pilotés par des hommes, mais véhiculent désormais les voix des femmes. En se voulant "l'écho fidèle de leurs approbations, de leurs critiques, de leurs justes revendications ${ }^{20}$ ", La Fronde, journal républicain en contact étroit avec les groupes socialistes, occupe de façon plutôt radicale une place singulière, notamment par la composition de l'équipe rédactionnelle exclusivement dirigée, administrée et composée de femmes ${ }^{21}$.

L'austérité typographique assumée dans ses pages, parfaitement adaptée au sérieux des thématiques souvent abordées, " contamine " également la rubrique artistique et culturelle. L'amélioration du sort des femmes dans le domaine artistique demeure par exemple au centre des préoccupations de ce journalisme militant permettant à plusieurs auteures de s'engager sur ces sujets brûlants. Le journal L'Abeille. Féministe internationale, dirigé par Pauline Savari, sert entre autres de tribune à la défense d'un syndicalisme pour la catégorie intermédiaire, souvent minorée et mal considérée, des femmes œuvrant au sein des arts décoratifs et appliqués ${ }^{22}$. La Fronde offre aussi à Harlor (alias Jeanne-Fernande Perrot) la possibilité de dénoncer, en 1904, l'absence de femmes dans les comités de la Société nationale des beaux-arts comme dans celui du Syndicat de la Presse artistique ${ }^{23}$, et à Marguerite Durand, l'année suivante, d'interpeller les dirigeants de l'École des beaux-arts pour empêcher la limitation du nombre de femmes au concours d'entrée ${ }^{24}$.

19. Marcelle Tinayre, « Ménages d'artistes », La Fronde, n 85, 3 mars 1896, p. 1.

20. [Marguerite Durand], La Fronde, n 3, 11 décembre 1897, p. 4.

21. Voir Mary Louise Roberts, "Copie subversive : le journalisme féministe en France à la fin du siècle dernier », Clio. Histoire, femmes et sociétés, n 6, 1997, p. 230-249.

22. Voir [Anonyme], "Les arts de la femme. Première exposition des femmes peintres en miniature sur émaux, porcelaines, éventails, etc. », L'Abeille. Féministe internationale, n 1, janvier 1901.

23. Harlor, « Les femmes au Salon de la Société nationale », La Fronde, n²100, 17 avril 1904.

24. Marguerite Durand, « Les Femmes aux beaux-arts », La Fronde, n²109, 16 février 1905. 
Parallèlement à ces revendications sociopolitiques qui témoignent bien - par l'usage fréquent du pseudonyme - d'un engagement sans précédent de la part des femmes, les journaux féministes jouent un rôle incontestable dans la lutte contre les clichés en proposant une image nouvelle des créatrices, principalement diffusée par l'intermédiaire d'une critique d'art féministe de plus en plus affirmée.

Les textes de Léonie Mulier (La Femme dans la famille et dans la société et La Femme et l'enfant) et d'Harlor (La Fronde) sont certainement parmi les plus engagés. Farouchement attachées à l'idée de défendre une image " autre » de la femme artiste, focalisée sur la qualité de ses productions et sur son statut professionnel, ces critiques rehaussent le statut de la femme artiste en mettant en avant ses compétences et son talent. Ainsi, face au nombre grandissant d'artistes femmes exposant dans les Salons, Léonie Mulier soutient, en 1884, dans La Femme et l'enfant " qu'à aucune époque il n'a été vu un mouvement emportant vers la même idée d'art un aussi grand nombre d'aptitudes et d'intelligences $^{25}$ ". De son côté, dans son article de 1905 paru dans La Fronde, Harlor incrimine davantage encore les discours ambiants, accoutumés à rabaisser les femmes, et n'hésite pas à lever le voile sur la trop grande médiocrité de certaines œuvres féminines, afin de les faire évoluer et de les hisser à un niveau de qualité comparable à celui des hommes ${ }^{26}$.

Ces exemples révèlent ainsi l'émergence d'une critique d'art féministe qui prend le contre-pied de la critique d'art dominante, traditionnellement écrite par des hommes et marquée par une tendance à reléguer les femmes artistes au rang d'aimables dilettantes. Désormais, le but est de mettre en lumière la qualité et la diversité des productions réalisées par certaines créatrices.

Dans ce contexte militant qui voit poindre une forme de démocratisation du jugement artistique, la critique Léonie Mulier radicalise son point de vue en inaugurant une chronique artistique dans la revue progressiste La Femme dans la famille et dans la société, où elle s'insurge contre le sort réservé aux femmes artistes et choisit de ne s'intéresser qu'aux œuvres exclusivement produites par les femmes ou traitant plus ou moins directement des femmes :

Le Salon ouvre ses portes le $1^{\text {er }}$ mai, telle est l'annonce pleine de promesses que vous avez pu lire dans tous les journaux. Toute la presse donnera des comptes rendus du Salon. La Femme ne restera pas en arrière. Comme l'on doit s'y attendre, ce compte rendu sera fait à un point de vue spécial : nos confrères ne s'occupent

25. Léonie Mulier, «Le Salon de 1884 », La Femme et l'enfant. Revue du progrès social et des questions $d^{\prime}$ enseignements, $n^{\circ} 2,1884$, p. 78-79.

26. Harlor, « Le Salon des Femmes peintres et sculpteurs », La Fronde, n²109, 16 février 1905. 
des artistes femmes qu'incidemment : nous, nous en ferons le but de nos critiques. Nos articles seront généralement divisés en trois parts, inégales il est vrai : la première sera consacrée aux œuvres féminines, la seconde aux portraits et aux tableaux représentant des penseurs qui se sont occupés de la femme, ou des scènes de l'histoire où elle a joué un rôle prépondérant ; enfin la troisième part sera consacrée aux jeunes, car nous ne devons ni ne pouvons oublier que nous sommes dans leurs rangs.

Aux personnes qui trouveront cette manière de voir bien partiale, je répondrai en changeant quelque peu un mot célèbre : "Je ne nie pas l'Homme, j'affirme la Femme." ${ }^{27}$.

Mulier développe par là, non seulement un discours esthétique mais également un discours politique, voire théorique, où les femmes critiques d'art, avant d'être commentatrices, se revendiquent d'abord femmes et spectatrices. Son regard alternatif et actif brouille les repères pour mieux interroger le hiatus entre réalité et fiction, dehors et dedans, femmes rêvées et femmes réelles ${ }^{28}$.

La volonté de mettre en avant un point de vue spécifiquement féminin se retrouve également à cette époque dans la constitution théorique même de l'art, au cour par exemple de l' "essai d'esthétique féminine " proposé par Clothilde Dissard en 1895 dans La Revue féministe ${ }^{29}$. Postulant une "psychologie des sexes ", cet article participe aux mutations que connaissent au XIX ${ }^{e}$ siècle les théories esthétiques avec l'essor de la psychologie de l'art ${ }^{30}$. Ayant pour objectif de fonder scientifiquement le savoir, ces traités esthétiques s'imprègnent de psychologie, pour expliquer les effets que l'art est à même de susciter par ses moyens formels. Dissard prolonge alors une tradition historique des doctrines sur l'art et de ses débats en réveillant les querelles anciennes du dessin et de la couleur associés respectivement au masculin et au féminin. Dans la lignée d'Aristote qui oppose dans La Génération des animaux une dualité du masculin associé à la forme et un féminin reposant plutôt sur la matière, les querelles entre poussinistes et rubénistes au XVII ${ }^{\mathrm{e}}$ siècle, entretenues au XIX ${ }^{\mathrm{e}}$ siècle notamment entre la ligne ingresque et le coloris delacrucien, ont maintenu ce

27. Léonie Mulier, "Le Salon », La Femme dans la famille et dans la société. Journal littéraire et scientifique, paraissant le samedi, $n^{\circ} 3,7$ mai 1880, p. 6.

28. Voir Laurence Brogniez, « Les femmes au Salon... », art. cité, p. 125.

29. Clotilde Dissard, « Essai d'esthétique féminine », La Revue féministe, 1895, p. 10-12.

30. Ibid., p. 10 : "La psychologie des sexes s'éclaire d'une lumière très vive par la comparaison de l'idéal masculin et de l'idéal féminin, par l'étude des causes de leurs divergences. » Pour une étude approfondie de cette question, voir Jacqueline Lichtenstein, Carole Maigné, Arnauld Pierre (dir.), Vers la science de I'art. L'esthétique scientifique en France (1857-1937), Paris, Presses de I'université Paris-Sorbonne, 2013. 
système de polarités associant le dessin au savoir intellectuel et le coloris à la sensualité et à la trivialités1.

Réceptive à ces discours qui ont forgé une hiérarchisation des procédés techniques au sein même de la peinture, Dissard reconduit ce qu'elle appelle le "partage des qualités esthétiques ", en expliquant que "[p]lus peut-être que par l'exquise simplicité de l'organisme, l'âme féminine est charmée par le brillant du coloris, la délicatesse des nuances, la chaude harmonie des teintes, la discrétion et la subtilité des parfums $»^{32}$. Convoquant l'inconstance et le changement propres - selon elle - à la psychologie féminine, la critique conclut en opposant très conventionnellement une esthétique du mouvement assortie au féminin à une esthétique de la forme propre au masculin ${ }^{33}$. Elle annonce par là ce que Guillaume Apollinaire expliquera en 1912 au sujet de la danseuse Loïe Fuller, " cette grande artiste de la ligne et des couleurs [...] qui fut le précurseur de l'art féminin d'aujourd'hui quand elle inventa cette chose géniale où se mêlaient la peinture, la danse, le dessin et la coquetterie et que l'on appela très justement : la danse serpentine ${ }^{34} »$.

Les auteures de presse féministe cherchent surtout à extirper les femmes artistes du carcan dans lequel on a bien souvent voulu les enfermer. Elles affirment aussi une identité et une individualité qui leur seraient désormais propres, sans pour autant, cela va sans dire, être inférieures. Ces journalistes renversent là, sans nul doute, les conventions sociales, et agissent comme de précieux soutiens dans la reconnaissance, la visibilité et la légitimité des œuvres et des carrières féminines.

À ce titre, il n'est pas inutile de rappeler que ces discours, déterminants pour la professionnalisation et la reconnaissance des femmes dans l'art, se situent sous la Troisième République, période marquée par une quête de justice sociale et de développement conjoint du féminisme et du socialisme, ou plutôt des féminismes et des socialismes. Les écrits de ces journalistes engagées puisent amplement dans les théories de lutte des classes défendues par

31. Voir, à ce sujet, Jacqueline Lichtenstein, "Le conflit du coloris et du dessin », dans La Couleur éloquente. Rhétorique et peinture à l'âge classique, Paris, Flammarion, 1989, p. 169-182 ; Georges Roque, "Portrait de la couleur en femme fatale », Art \& Fact, n 10, 1991, p. 4-11; Patricia L. Reilly, "Writing out Colour in Renaissance Theory », Genders, n 12, 1991, p. 77-99 ; Jean-Pierre Guillerm, "La couleur et le féminin dans la critique picturale de la seconde moitié du xix siècle », dans Jean-Loup Korzilius (dir.), Couleur de la morale. Morale de la couleur, Besançon, Presses universitaires de Franche-Comté, 2010, p. 49-58.

32. Clotilde Dissard, « Essai d'esthétique féminine », art. cité, p. 11-12.

33. Ibid.

34. Guillaume Apollinaire, "Chroniques d'art. Les peintresses », Chroniques d'art 1902-1918, Paris, Gallimard, 1993 [Le Petit Bleu, 5 avril 1912], p. 307. 
Karl Marx, August Bebel ou encore Friedrich Engels, qui, dans leurs écrits, considèrent les femmes comme des victimes de la civilisation bourgeoise et présentent leur participation au travail comme la condition nécessaire à leur autonomisation financière et à leur indépendance vis-à-vis des hommes ${ }^{35}$.

\section{Femmes artistes dans la presse féminine : une émancipation mitigée}

Face à un féminisme politique exhortant les femmes à sortir de l'espace domestique, se développe simultanément un féminisme en demi-teinte véhiculé par de luxueux magazines féminins illustrés. Deux d'entre eux, Femina et La Vie heureuse, fondés par des hommes mais destinés à un lectorat féminin, participent à ce "féminisme mystifié " pour reprendre la formule de Colette Cosnier $^{36}$.

L'artiste, à côté de la romancière, y est érigée en véritable modèle émancipateur soutenu par des titres aussi bruyants que «Une femme remporte le prix de Rome " (ill. $\mathrm{n}^{\circ} 1$ ) ou "Le triomphe de l'art féminin ${ }^{37}$. Les mises en page illustrent avec précision une nouvelle individualité conquise par les femmes artistes qui parviennent à dépasser le clivage création/procréation, pour combiner harmonieusement les deux. Le genre du reportage, privilégié dans ces articles, contribue à fabriquer un portrait sur le vif de l'artiste au quotidien. Loin de s'attarder sur leur production, c'est plutôt sur l'image de leurs auteures que ces magazines se focalisent et qui, même s'ils ne s'engagent pas véritablement dans les débats sociaux contemporains, doit leur permettre d'illustrer la modernisation d'une société en pleine mutation ${ }^{38}$.

35. Voir à ce sujet, Charles Sowerwine, "Marx, Bebel, and Engels on the Woman Question », Sisters or Citizens? Women and Socialism in France since 1876, Cambridge, Cambridge University Press, 2008 [1982], p. 15-18. Rapidement diffusé et traduit en France à la fin du siècle, La Femme et le Socialisme d'August Bebel, paru pour la première fois en 1879, analyse la condition économique et sexuelle de I'ouvrière en reprenant les principaux arguments du Capital de Karl Marx. Seule la suppression de la dépendance économique peut, selon lui, affranchir la femme de la tutelle masculine. II engage donc à lutter au sein du prolétariat pour fonder de nouvelles relations entre les sexes. De son côté, Friedrich Engels explique, dans L'Origine de la famille, de la propriété privée et de l'État (1884), que la première division du travail est celle entre l'homme et la femme et que, de là, est née l'oppression de l'homme sur la femme.

36. Colette Cosnier, Les Dames de Femina. Un féminisme mystifié, Rennes, Presses universitaires de Rennes, 2009, 308 p.

37. H. A., " Une femme remporte le prix de Rome », Femina, n² 254, 15 août 1911, p. 446 ; voir Nicole Odry, « Le Triomphe de l'art féminin », Femina, n 173, $1^{\text {er }}$ avril 1908, p. 145-146.

38. Voir Hélène Eck, Claire Blandin, "Devoirs et désirs : les ambivalences de la presse féminine », dans Hélène Eck et Claire Blandin (dir.), La Vie des femmes, op. cit., p. 8. 


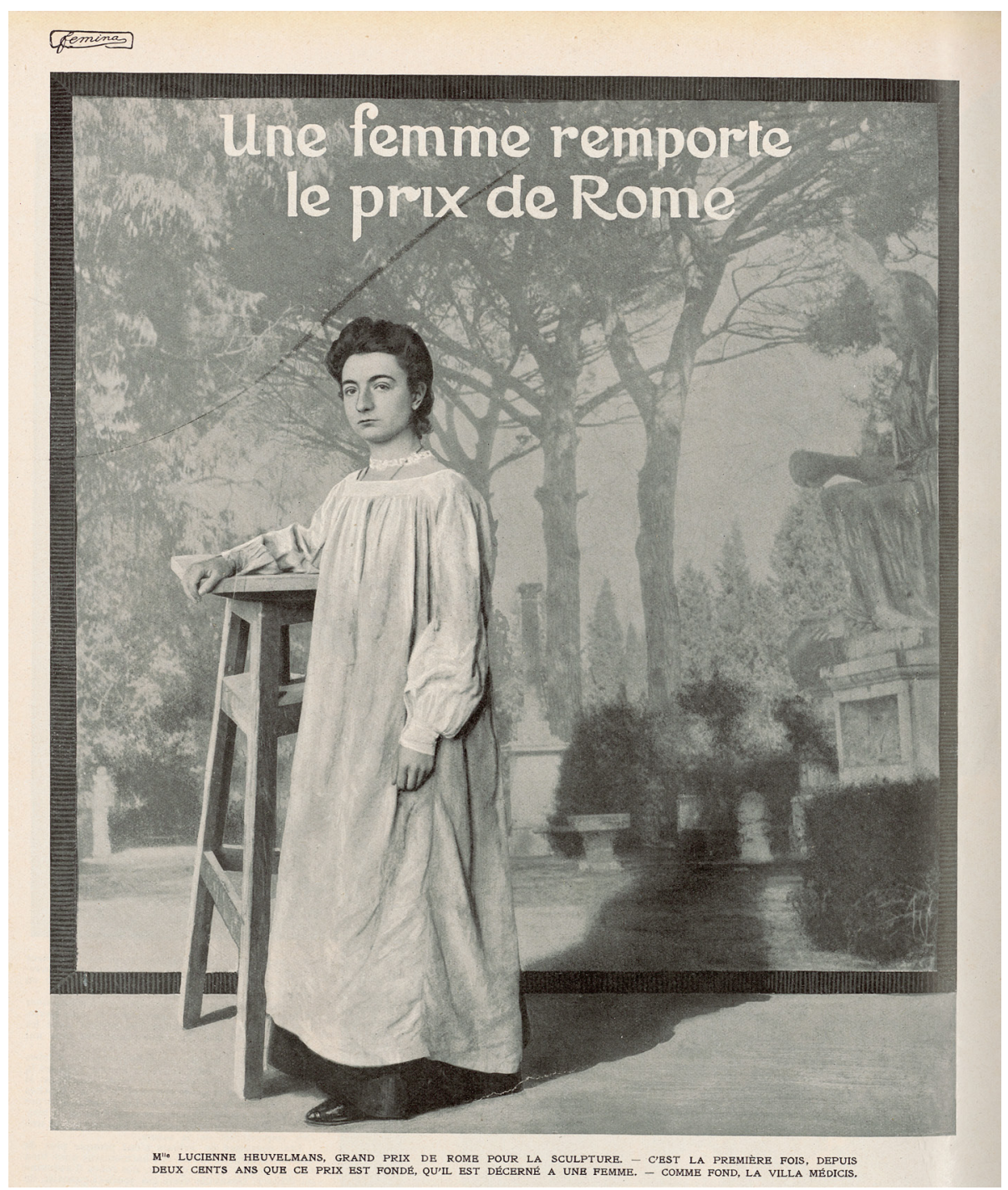

Ill. 1 - H. A., «Une femme remporte le prix de Rome », Femina, n² 254, 15 août 1911, p. 446 (C) droits réservés

Un article de La Vie heureuse, paru en 1904 présente ainsi la peintre Clémentine-Hélène Dufau en icône de mode élégante ${ }^{39}$. Un autre article de

39. [Anonyme], «Mademoiselle Dufau », La Vie heureuse, n 3, mars 1904, p. 56 : " L'auteur est une jeune femme. Elle a le teint mat avec des yeux sombres; la figure et la taille élégantes; des cheveux, qu'elle tord en une coque haute, légère et noire. » 
Femina fait de Camille Claudel une créature mythifiée surprenant par son labeur comme par le pouvoir presque fatal de sa beauté :

Quai de Béthune, dans ce coin pittoresque et seigneurial du vieux Paris, dans le décor paisible de la Seine, qui traîne avec elle l'odeur des pommes, emplissant les bateaux, $\mathrm{M}^{\text {Ile }}$ Claudel travaille à son envoi du Salon.

[...]

- Ce travail est dur! Si le modelage de la glaise est déjà fatiguant pour une femme, que dire de ce travail, à même le marbre. Vous devez être la seule, Mademoiselle, à oser tailler la matière ?

[...]

$\mathrm{M}^{\text {Hle }}$ Claudel sourit, ses yeux se tournent vers moi, d'un vert pâle, qui évoque les jeunes pousses des fortes. Ces yeux surprennent par leur clarté, ils ont un charme virgilien, puisqu'ils évoquent tout de suite la fraîcheur des bois. Mais au moment même où le regard vous attire, un geste instinctif de l'artiste semble arrêter l'élan de sa sympathie, et l'on reste avec cette impression bizarre d'une nature profondément personnelle, qui vous attire par sa grâce et vous repousse par sa sauvagerie. Tout le caractère de $\mathrm{M}^{\mathrm{lle}}$ Claudel est dans ce retrait un peu farouche ${ }^{40}$.

Particulièrement révélateurs, ces exemples témoignent non seulement d'une certaine volonté de développer une image rassurante de la femme artiste en modèle attractif, puisqu' elle parvient à pénétrer la sphère du masculin tout en préservant ses attraits féminins ${ }^{41}$, mais rejoignent également le clivage peinture/ sculpture, où quand la femme peintre est un être charmant et élégant, la sculptrice s'associe davantage à la virilité. Paul Gilbert précise, en ce sens, que la peinture permet aux femmes de développer leurs qualités de délicatesse et d'habileté et qu'elle convient parfaitement " à [leur] organisation, à [leur] genre de vie ${ }^{42} »$. Au contraire, la sculpture semble lui être inadaptée comme l'explique Octave Uzanne ${ }^{43}$. De même, Paul Claudel écrit plus radicalement dans le catalogue de l'exposition posthume dédiée à sa sœur en 1951, et en gardant certainement en tête les dernières années recluses de l'artiste, que « [1]e métier de sculpteur est pour un homme une espèce de défi perpétuel au bon

40. M. Rivière, "Les Artistes Femmes au Salon de 1903 », Femina, n 53, $1^{\text {er }}$ avril 1903, p. 520-521.

41. Cette idée est au cœur même de la revue et de son programme. Voir Pierre Lafitte, Femina, $n^{\circ} 1$,

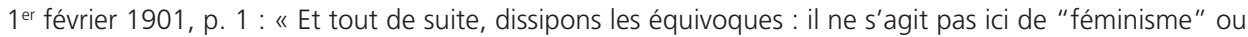
"d'émancipation féminine" : nous laissons à d'autres le soin de masculiniser la femme et de lui enlever son charme exquis. Femina sera, au contraire à la vraie femme, à la Française élevée sainement dans les meilleures traditions d'élégance, de bon ton et de grâce. »

42. Paul Gilbert, "Les femmes artistes », La Jeune fille. Journal hebdomadaire paraissant le jeudi dirigé par des femmes du monde, $n^{\circ} 3,19$ juillet 1888, p. 37.

43. Octave Uzanne, La Femme à Paris. Nos contemporaines. Notes successives sur les Parisiennes de ce temps dans leurs divers milieux, états et conditions, Paris, Ancienne maison Quantin, 1894, p. 163. 
sens [mais] pour une femme isolée et pour une femme du tempérament de ma sœur une pure impossibilitét ${ }^{4}$ ». L'historienne de l'art Marjan Sterckx a notamment démontré, qu'en plus des obstacles physiques, économiques et culturels qui rendent pour les femmes difficiles - mais pas forcément insurmontables l'exercice de cet art, la sculpture suppose des pratiques qui sont elles-mêmes genrées $^{45}$. Ainsi, l'exercice de la taille directe impliquant le travail de matériaux durs et difficiles, comme la pierre ou le bois, nécessaires à la réalisation de sculptures publiques ou monumentales, serait plutôt du côté masculin quand la technique du modelage favorisant l'emploi de matériaux souples et plus malléables (argile, cire), pour des sculptures de petites dimensions situées dans des espaces privés et domestiques, serait plus appropriée aux femmes.

Alors que la personnalité et le quotidien de ces artistes présentées comme travailleuses et émancipées sont mis en lumière de façon inédite et a priori positive, leur valorisation dissimule, si l'on regarde de plus près les textes et les légendes, un propos plus conventionnel qui reconduit les clichés et rabat inévitablement la femme dans la sphère domestique. Participant à ce "féminisme familial ${ }^{46}$ ", Marthe Boyer-Breton rappelle par exemple, dans un article de Femina paru en 1905, qu'il est intéressant d'analyser " ce mélange d'aspirations artistiques et de préoccupations familiales qui se rencontre chez toute femme-peintre ${ }^{47}$ ». La femme prédomine alors sur l'artiste, contrainte, quant à elle, de plier devant l'ordre social et de gouverner la sphère familiale :

La femme - tant pis si je parais plaider pro domo - est moins favorisée. Tout artiste qu'elle soit, n'est-elle pas femme d'abord ? Femme, c'est-à-dire soumise à mille obligations naturelles ou sociales, obligée aux soins de son foyer, de ses enfants, de son mari, et même astreinte à certaines servitudes mondaines. Son atelier est une pièce du logis familial : des marmots y jouent parfois - et de manière combien pendable - dans un coin, tandis que la maman tâche à fondre une teinte, à animer un geste, à aviver une expression ou à l'adoucir ; la bonne vient l'y consulter sur le menu du dîner, une amie, "en passant" - c'est toujours en passant que nos amies nous dérangent -, vient troubler de son babillage le rêve évoqué, empêche

44. Paul Claudel, Camille Claudel. Décembre 1864 - octobre 1943, catalogue d'exposition, Paris, Musée Rodin, novembre-décembre 1951, Paris, Aulard, 1951, p. 7-8.

45. Marjan Sterckx, «Pride and Prejudice. Eighteenth-Century Women Sculptors and their Material Practices », dans Jennie Batchelor et Cora Kaplan (dir.), Women and Material Culture 1660-1830, Londres/ New York, Palgrave Macmillan, 2007, p. 86-102.

46. Karen Offen, « Depopulation, Nationalism, and Feminism in Fin-de-Siècle France », American Historical Review, n89, 1984, p. 657.

47. Marthe Boyer-Breton, « La journée d'une femme-peintre », Femina, n 98, 15 février 1905, p. 91. 
d'achever sur l'heure le morceau entrepris! Ah! elle est bien remplie la journée d'une femme-peintre-mère-de-famille ${ }^{48}$.

S'adossant majoritairement à des stéréotypes, le féminisme en demi-teinte diffusé dans ces magazines provient également du corpus de femmes artistes choisi et mis en lumière. Loin d'être autodidactes ou issues de cercles artistiques alternatifs, ces artistes-là exposent dans les Salons officiels, reçoivent des récompenses, et sont le plus souvent saluées et reconnues par la critique. On croise d'ailleurs dans les milieux parisiens et mondains Charlotte Besnard, Clémentine Dufau, Louise Abbéma ou Madeleine Lemaire... et c'est bien elles que Femina et La Vie heureuse retiennent dans leurs colonnes.

En cherchant à poser les bases d'une "vie heureuse ", la presse féminine fournit donc à ses lectrices le modèle d'une femme rassurante, socle et pilier du foyer mais aussi une image vectrice d'idéalisation et de valorisation. Libérée des conventions sociales comme des préjugés biologiques, cette femme intrépide et moderne, reconnue socialement et épanouie professionnellement, incarne de fait le différentialisme proudhonien où si «l'homme est principalement une puissance d'ACTION ; la femme [incarne] une puissance de fascination ${ }^{49}$ ».

Colette Cosnier et Rachel Mesch expliquent ainsi que Femina et La Vie heureuse fonctionnent sur cette frontière presque impénétrable entre ces femmes exceptionnelles qu'incarnent les artistes ou les écrivaines, et les lectrices qui sont principalement des femmes du monde, révélant par là la profonde difficulté à définir le genre féminin comme une entité homogène. Ces images de femmes artistes, hors normes et inaccessibles, agissent comme des modèles utopiques, sortes de fabrications culturelles qui attirent le lectorat par un idéal de perfection tout en lui permettant de prendre conscience, de manière apaisée et intime, des mutations sociales et politiques que connaît au même moment le sort des femmes ${ }^{50}$.

Si les femmes artistes deviennent, en 1900, un support de fantasmes et d'idéologies, les femmes critiques d'art pourraient incarner tout autant la figure de l' " éclaireuse ». Analysée par Mary Louise Roberts dans son ouvrage sur la femme nouvelle ${ }^{51}$, ce personnage apparaît, effectivement, au début du

48. Marthe Boyer-Breton, "La journée d'une femme-peintre », art. cité, p. 92.

49. Pierre-Joseph Proudhon, La Pornocratie ou Les Femmes dans les Temps Modernes, Paris, Librairie internationale, 1875, p. 44

50. Voir Colette Cosnier, Les Dames de Femina, op. cit., p. 54 ; Rachel Mesch, Having It All in the Belle Epoque. How French Women's Magazines Invented the Modern Woman, Stanford, Stanford University Press, 2013, $241 \mathrm{p}$

51. Voir Mary Louise Roberts, Disruptive Acts. The New Woman in Fin-de-Siècle France, Chicago/Londres, University of Chicago Press, 2002, p. 39. 
$\mathrm{xx}^{\mathrm{e}}$ siècle, entre autres sous la plume de Maurice Donnay, pour désigner celle qui ouvre la voie du féminisme aux autres ${ }^{52}$. Et, comme pour consolider le dialogue entre femmes, plusieurs artistes n'hésitèrent également pas, de leur côté, à rendre hommage à ces pionnières. Amélie Beaury-Saurel, par exemple représente dans un tableau plusieurs femmes dont une artiste peintre à l'arrière-plan, tenant un pinceau, et une écrivaine accoudée sur une pile de livres, regroupées autour d'une avocate symbolisant la justice, le doigt pointé sur un livre de loi (ill. $n^{\circ} 2$ ). Si les deux femmes les plus proches de cette « justicière " semblent faire les frais d'une masculinisation troublante - il semble s'agir de l'archéologue Jane Dieulafoy et de la romancière Lucie Delarue-Mardrus, au fond, dans l'ombre - reconduisant les discours normatifs sur l'hypervirilisation de la femme prétendant à l'érudition, celles dévoilées par Clémentine-Hélène Dufau (ill. $\mathrm{n}^{\circ}$ 3) concilient plus positivement émancipation et féminité. Dans cette affiche réalisée sur commande pour La Fronde, une jeune femme rousse portant une robe verte - sans doute une journaliste, peut-être Marguerite Durand comme l'avance Ruth E. Iskin ${ }^{53}$ - montre le chemin à d'autres femmes, jeunes ou âgées, mères ou célibataires, travailleuses ou miséreuses, situées derrière elle, et dont l'avenir et l'espoir d'intégration dans la cité sont symbolisés par une ville en pleine mutation, tout juste éclairée par le lever du soleil ${ }^{54}$.

52. Maurice Donnay, Les Éclaireuses. Pièce en quatre actes, Paris, 13, rue Saint-Georges, 1913, 38 p.

53. Ruth E. Iskin, "Popularising New Women in Belle Époque Advertising Posters », dans Diana Holmes, Carrie Tarr (dir.), A "Belle Époque"? Women in French Society and Culture 1890-1914, New York/Oxford, Berghahn Books, 2006, p. 98.

54. Clémentine-Hélène Dufau et Amélie Beaury-Saurel sont formées à l'académie Julian. Beaury-Saurel y dirige d'ailleurs l'atelier des femmes à la mort de Rodolphe Julian, son époux, en 1907. Beaury-Saurel et Dufau exposent également aux Salons (Salon des artistes français, Salon d'Automne...). Parmi les œuvres de Beaury-Saurel qui peuvent témoigner d'un certain engagement politique, notons le portrait qu'elle réalise de la journaliste féministe Séverine (1893, huile sur toile, Paris, musée Carnavalet), ainsi que Dans le bleu (1894, pastel, Toulouse, musée des Augustins) où elle renouvelle le motif de la fumeuse en proposant une image rêveuse et mélancolique d'une femme fumant, assise à une table devant un café. Le décor totalement dépouillé peut, dans cette œuvre, créer une certaine ambiguïté sur le lieu de l'action et sur l'identité de cette femme, à savoir une épouse dans un intérieur domestique ou une femme seule dans un café. Clémentine-Hélène Dufau réalise une affiche au journal La Fronde sur la montée du féminisme en France, et expose à l'Union des Femmes Peintres et Sculpteurs. Mais sa renommée dépasse bientôt les seuls cercles féministes, et elle devient l'une des rares femmes au début $d u x x^{e}$ siècle à obtenir des commandes privées et publiques. Citons, par exemple, celle du dramaturge et poète Edmond Rostand qui lui demande, en 1909, de réaliser un décor monumental pour la bibliothèque de sa résidence basque à Cambo-les-Bains (Villa Arnaga), ou encore celle, plus officielle, de l'État pour la Salle des Autorités de la Sorbonne où elle réalise quatre panneaux monumentaux sur le thème des disciplines scientifiques. 


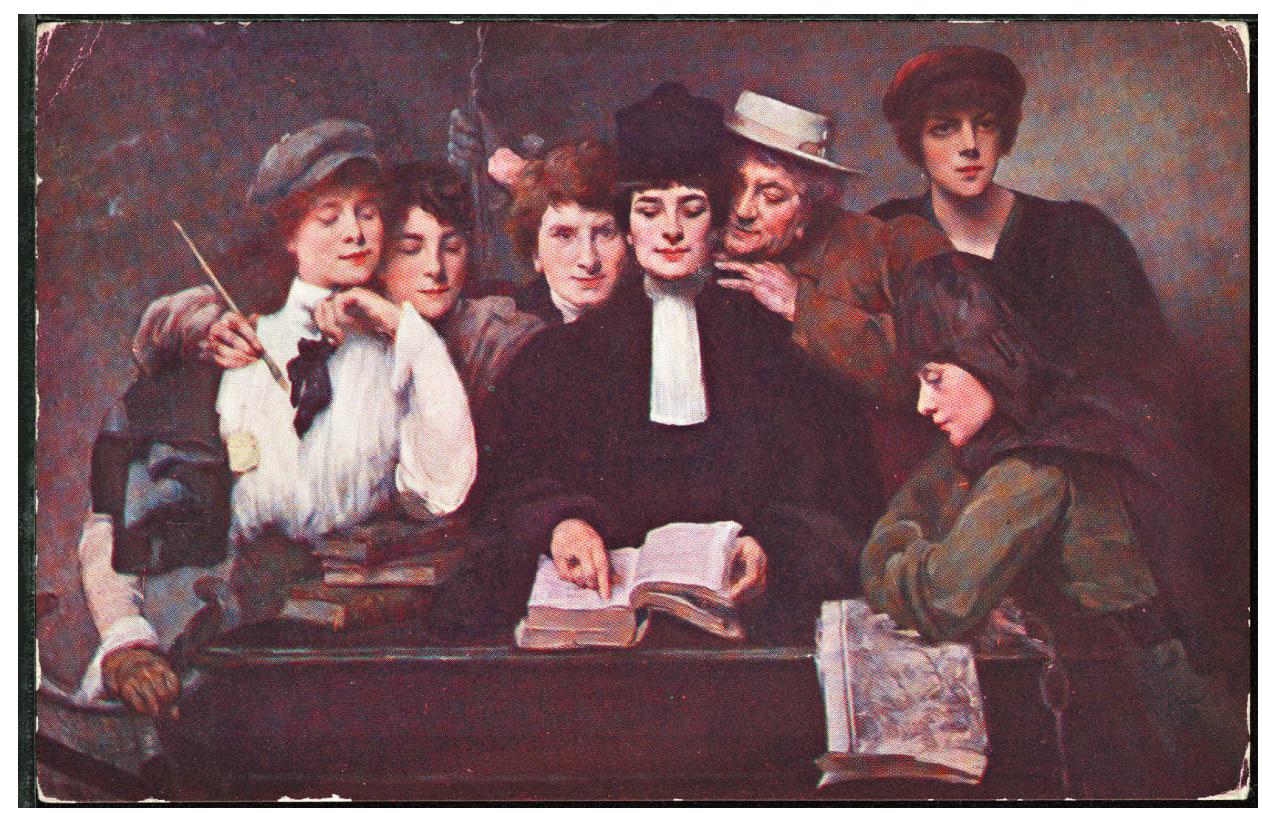

Ill. 2 - Amélie Beaury-Saurel, Nos éclaireuses, avant 1914, huile sur toile, dimensions inconnues, localisation actuelle inconnue.

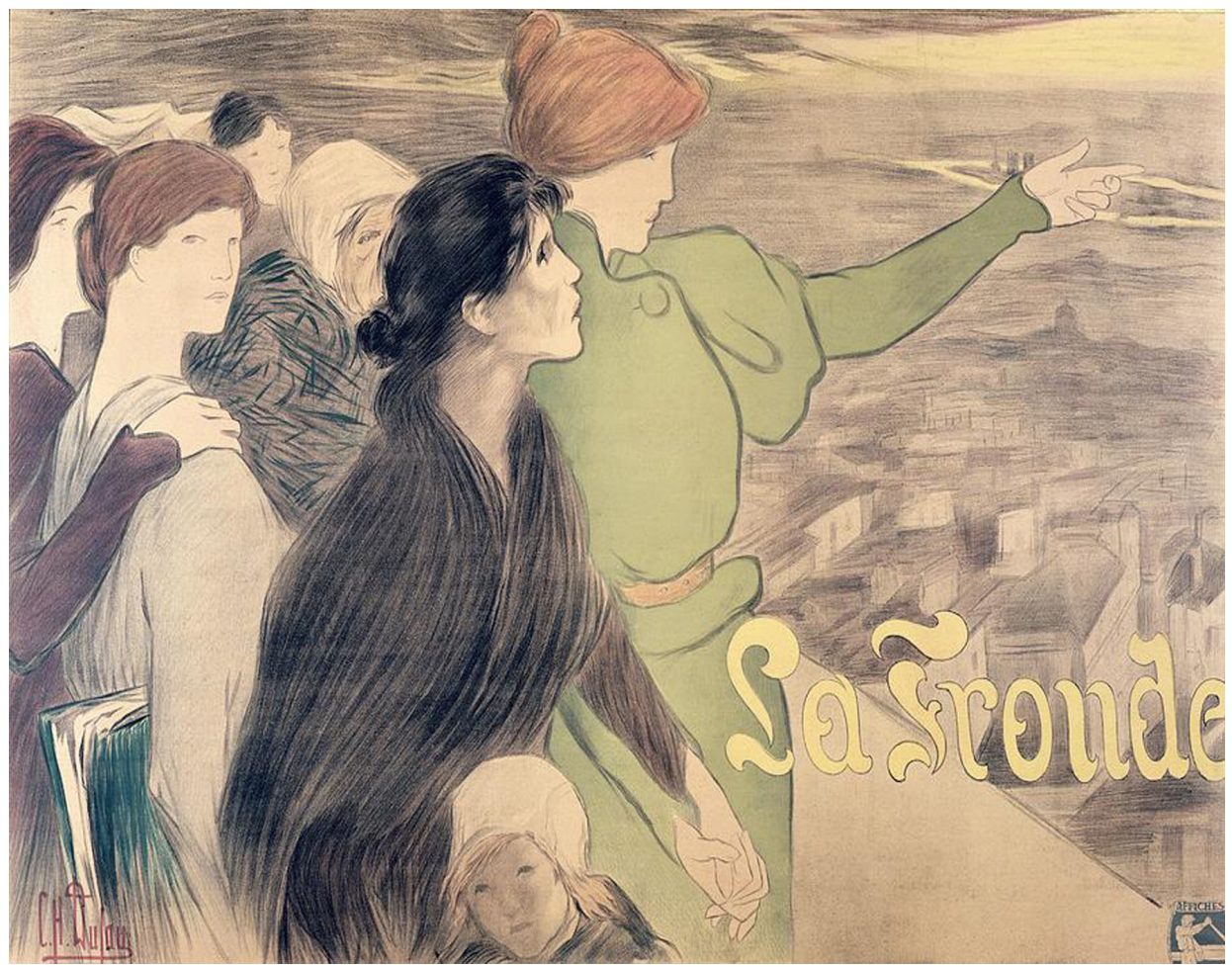

Ill. 3 - Clémentine-Hélène Dufau, La Fronde, 1898, chromolithographie, 99, 80 x 137, 80 cm, collection privée (C) droits réservés.

Charlotte Foucher Zarmanian, "Les femmes artistes sous presse »,

S. \& R., n40, automne 2015, p. 111-127. 
Dans ces deux œuvres, les éclaireuses s'incarnent aussi bien dans les femmes de loi que dans les journalistes ou les artistes - véritables citoyennes au sens large. De ce fait, ne pourrait-on pas envisager ces deux représentations comme des manifestes sociaux et politiques? En montrant notamment des femmes émancipées qui peuvent être des artistes, ne permettent-elles pas d'entrevoir efficacement un glissement du discours écrit sur l'art dans le geste même de peindre, de créer ? Ces portraits collectifs, peints par des femmes appelant elles-mêmes à une certaine indépendance dans la création, pourraient ainsi achever le dialogue solidaire et fécond, entre praticiennes et critiques d'art du même sexe.

Dans les décennies suivantes, ce dialogue se prolonge de manière éloquente si l'on songe aux apports déterminants de la Française Aline DallierPopper ou de l'Américaine Lucy Lippard ${ }^{55}$. Fidèles à cette notion de sororité, ces critiques d'art actives dans les années 1970 perpétuent l'entreprise proposée par leurs pairs au tout début $\mathrm{du} \mathrm{xx}^{\mathrm{e}}$ siècle. Soutenant activement la création des femmes en les intégrant au maximum dans les expositions qu'elles organisent et les articles qu'elles publient, elles poursuivent la lutte contre les discriminations du marché de l'art et dénoncent le phallocentrisme à l'œuvre dans la fabrique de l'histoire de l'art publiant alors les premières monographies sur Eva Hesse, Louise Bourgeois ou encore Charlotte Calmis : une façon de montrer que l'artiste peut aussi se penser au « féminin » singulier.

55. Voir, entre autres, Fabienne Dumont, « Aline Dallier-Popper, pionnière de la critique d'art féministe en France », Critique d'art. Revue critique et bibliographique, n 31, printemps 2008, p. 110-116 ; Aline Dallier-Popper, Art, féminisme, post-féminisme - Un parcours de critique d'art, Paris, L'Harmattan, 2009 ; Beth Anne Lauritis, Lucy Lippard and the Provisional Exhibition. Intersections of Conceptual Art and Feminism, 1970-1980, thèse de doctorat, Los Angeles, université de Californie, 2009 ; Cornelia H. Butler, From Conceptualism to Feminism. Lucy Lippard's Numbers Shows, 1969-1974, Londres, Afterall Books, 2012. 